Acta Crystallographica Section E

Structure Reports

Online

ISSN 1600-5368

\section{$1 H$-Benzimidazole-2(3H)-thione}

\section{De-Cai Wang, ${ }^{\mathrm{a} *}$ Shan $\mathrm{Mi}^{\mathrm{a}}{ }^{\mathrm{a}}$ Wei $\mathrm{Xu},{ }^{\mathrm{a}}$ Liang Jiang ${ }^{\mathrm{a}}$ and Xin-Ming Huang ${ }^{b}$}

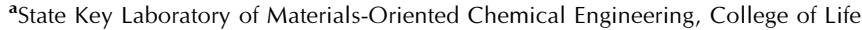
Science and Pharmaceutical Engineering, Nanjing University of Technology, Xinmofan Road No. 5 Nanjing, Nanjing 210009, People's Republic of China, and ${ }^{\mathbf{b}}$ College of Science, Nanjing University of Technology, Xinmofan Road No. 5 Nanjing, Nanjing 210009, People's Republic of China

Correspondence e-mail: dcwang@njut.edu.cn

Received 27 February 2009; accepted 5 March 2009

Key indicators: single-crystal X-ray study; $T=294 \mathrm{~K}$; mean $\sigma(\mathrm{C}-\mathrm{C})=0.004 \AA$; $R$ factor $=0.049 ; w R$ factor $=0.152 ;$ data-to-parameter ratio $=18.1$.

The asymmetric unit of the title compound, $\mathrm{C}_{7} \mathrm{H}_{6} \mathrm{~N}_{2} \mathrm{~S}$, contains one half-molecule; the $\mathrm{C}$ and $\mathrm{S}$ atoms of the $\mathrm{C}=\mathrm{S}$ group lie on a crystallographic mirror plane. In the crystal structure, intermolecular $\mathrm{N}-\mathrm{H}$...S hydrogen bonds link the molecules.

\section{Related literature}

For a related structure, see: Mavrova et al. (2007). For bondlength data, see: Allen et al. (1987).

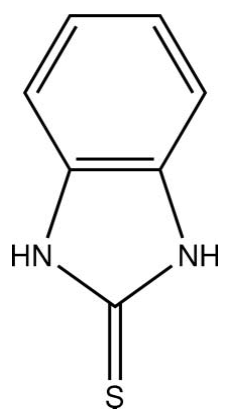

\section{Experimental}

\section{Crystal data}

$\mathrm{C}_{7} \mathrm{H}_{6} \mathrm{~N}_{2} \mathrm{~S}$

$M_{r}=150.21$

Monoclinic, $P 2_{1} / m$ $\beta=91.76(3)^{\circ}$

$V=348.66(12) \AA^{3}$

$Z=2$

Mo $K \alpha$ radiation

Data collection

Enraf-Nonius CAD-4 diffractometer

Absorption correction: $\psi$ scan (North et al., 1968)

$T_{\min }=0.896, T_{\max }=0.963$

903 measured reflections

Refinement

$R\left[F^{2}>2 \sigma\left(F^{2}\right)\right]=0.049$

$w R\left(F^{2}\right)=0.152$

$S=1.00$

813 reflections $\mu=0.38 \mathrm{~mm}^{-1}$

$T=294 \mathrm{~K}$

$0.30 \times 0.20 \times 0.10 \mathrm{~mm}$

813 independent reflections 647 reflections with $I>2 \sigma(I)$ $R_{\text {int }}=0.044$

3 standard reflections frequency: $120 \mathrm{~min}$ intensity decay: $1 \%$

45 parameters

$\mathrm{H}$-atom parameters constrained

$\Delta \rho_{\max }=0.37{\mathrm{e} \AA^{-3}}^{-3}$

$\Delta \rho_{\min }=-0.26 \mathrm{e} \AA^{-3}$

Table 1

Hydrogen-bond geometry $\left(\AA,^{\circ}\right)$.

\begin{tabular}{lllll}
\hline$D-\mathrm{H} \cdots A$ & $D-\mathrm{H}$ & $\mathrm{H} \cdots A$ & $D \cdots A$ & $D-\mathrm{H} \cdots A$ \\
\hline $\mathrm{N}-\mathrm{H} 0 A \cdots \mathrm{S}^{\mathrm{i}}$ & 0.86 & 2.57 & $3.3798(19)$ & 158 \\
\hline
\end{tabular}

Symmetry code: (i) $-x, y-\frac{1}{2},-z+2$.

Data collection: CAD-4 Software (Enraf-Nonius, 1989); cell refinement: $C A D-4$ Software; data reduction: XCAD4 (Harms \& Wocadlo, 1995); program(s) used to solve structure: SHELXS97 (Sheldrick, 2008); program(s) used to refine structure: SHELXL97 (Sheldrick, 2008); molecular graphics: ORTEP-3 for Windows (Farrugia, 1997) and PLATON (Spek, 2009); software used to prepare material for publication: SHELXTL (Sheldrick, 2008).

The authors thank the Center of Testing and Analysis, Nanjing University, for support.

Supplementary data and figures for this paper are available from the IUCr electronic archives (Reference: HK2638).

\title{
References
}

Allen, F. H., Kennard, O., Watson, D. G., Brammer, L., Orpen, A. G. \& Taylor, R. (1987). J. Chem. Soc. Perkin Trans. 2, pp. S1-19.

Enraf-Nonius (1989). CAD-4 Software. Enraf-Nonius, Delft, The Netherlands.

Farrugia, L. J. (1997). J. Appl. Cryst. 30, 565.

Harms, K. \& Wocadlo, S. (1995). XCAD4. University of Marburg, Germany.

Mavrova, A. Ts., Denkova, P., Tsenov, Y. A., Anichina, K. K. \& Vutchev, D. I. (2007). Bioorg. Med. Chem. 15, 6291-6297.

North, A. C. T., Phillips, D. C. \& Mathews, F. S. (1968). Acta Cryst. A24, 351359.

Sheldrick, G. M. (2008). Acta Cryst. A64, 112-122.

Spek, A. L. (2009). Acta Cryst. D65, 148-155. 


\section{supporting information}

Acta Cryst. (2009). E65, o756 [doi:10.1107/S1600536809008058]

\section{H-Benzimidazole-2(3H)-thione}

\section{De-Cai Wang, Shan Mi, Wei Xu, Liang Jiang and Xin-Ming Huang}

\section{S1. Comment}

It is a kind of secondary age inhibitor, and could reinforce the effect combined with DNP AP and other nonpolluting age inhibitors. It disperses easily in rubber, and the color does not change under sun exposure. Its pollution capacity is limited. 2-Mercaptobenzimidiazole is a new kind of anti-leprosy drugs, and its toxicity is lower than sulphone drugs. It should not be used in the patients to which can not be given sulphone drugs. We report herein the crystal structure of the title compound.

The asymmetric unit of the title compound (Fig. 1) contains one-half molecule, in which a mirror plane passes through $\mathrm{S}$ and $\mathrm{C} 4$ atoms. The bond lengths (Allen et al., 1987) and angles are within normal ranges.

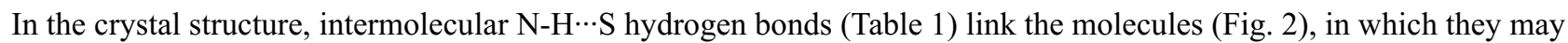
be effective in the stabilization of the structure.

\section{S2. Experimental}

For the preparation of the title comppund, 1,2-diaminobenzene $(0.019 \mathrm{~mol})$ and water $(3 \mathrm{ml})$ were added to a solution of sodium hydroxide $(0.022 \mathrm{~mol})$ in ethanol $(20 \mathrm{ml})$ and carbon disulfide $(0.022 \mathrm{~mol})$. The mixture was heated under reflux for $3 \mathrm{~h}$. Charcoal was added cautiously and removed by filtration after the mixture has been refluxed for 10 min more.

The filtrate was heated to $377 \mathrm{~K}$ and quenched with warm water $(377 \mathrm{~K}, 20 \mathrm{ml})$, and then acetic acid $(9 \mathrm{ml})$ was added by stirring. The product was separated and after cooling in refrigerator for $3 \mathrm{~h}$ the crystallization was completed (Mavrova et al., 2007). Crystals suitable for X-ray analysis were obtained by slow evaporation of an ethanol solution after two weeks.

\section{S3. Refinement}

$\mathrm{H}$ atoms were positioned geometrically, with $\mathrm{N}-\mathrm{H}=0.86 \AA$ (for $\mathrm{NH}$ ) and $\mathrm{C}-\mathrm{H}=0.93 \AA$ for aromatic $\mathrm{H}$ and constrained to ride on their parent atoms, with $\mathrm{U}_{\text {iso }}(\mathrm{H})=1.2 \mathrm{U}_{\mathrm{eq}}(\mathrm{C}, \mathrm{N})$. 


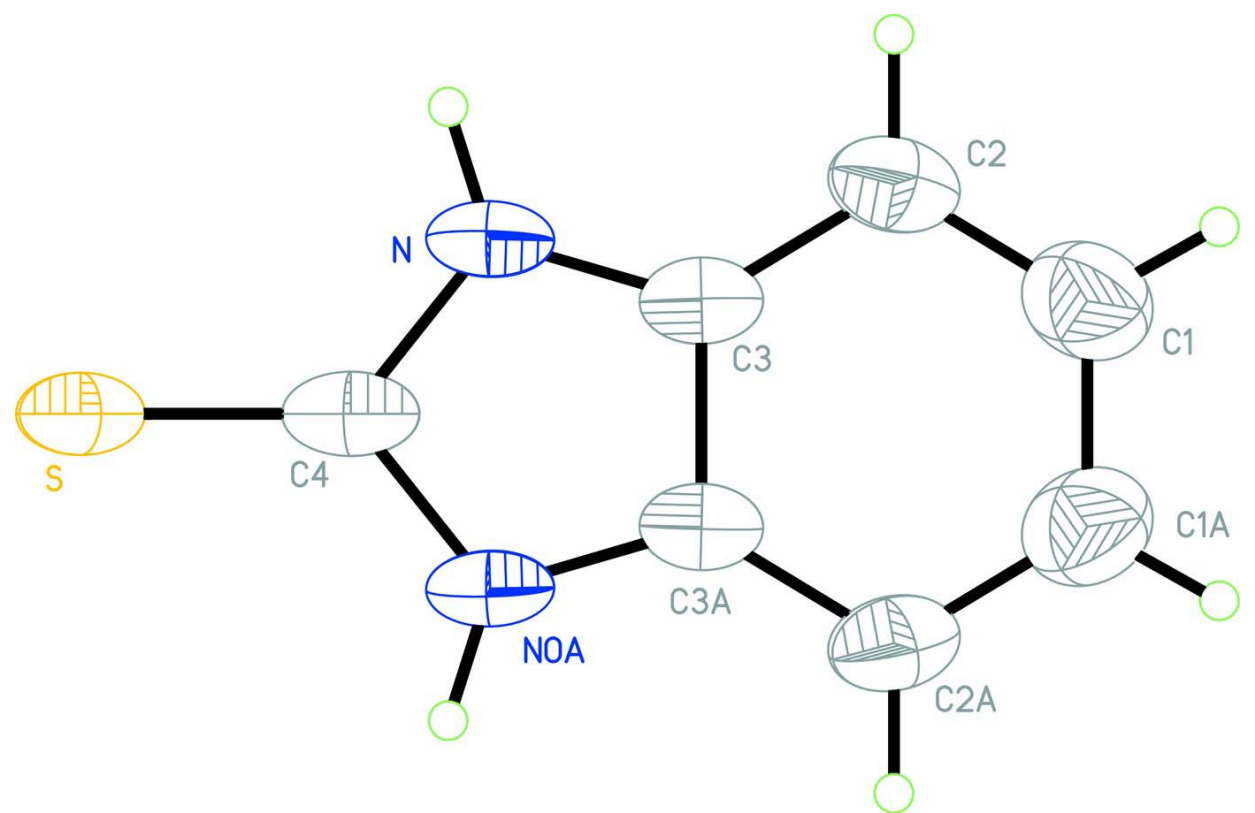

\section{Figure 1}

The molecular structure of the title molecule, with the atom-numbering scheme. Displacement ellipsoids are drawn at the $30 \%$ probability level.

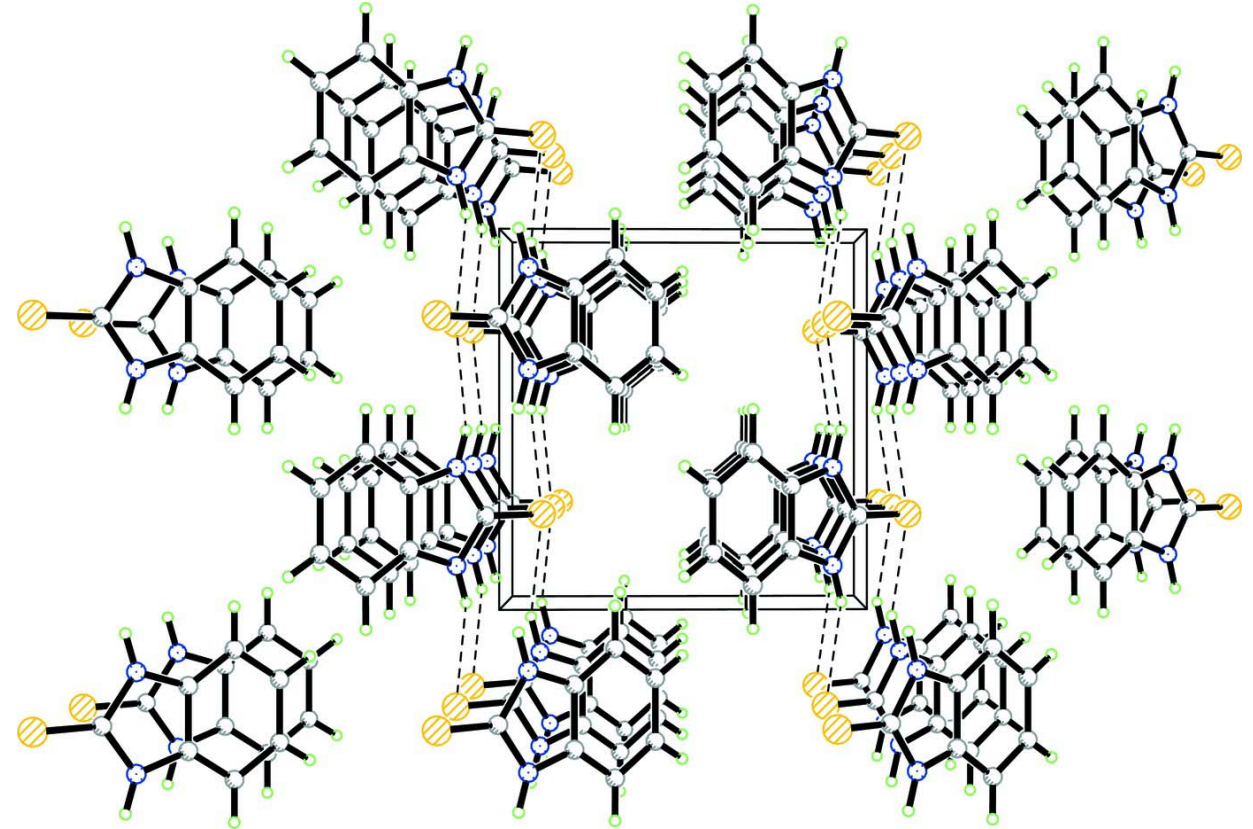

Figure 2

A partial packing diagram. Hydrogen bonds are shown as dashed lines. 


\section{$1 H$-Benzimidazole-2(3H)-thione}

Crystal data

$$
\begin{aligned}
& \mathrm{C}_{7} \mathrm{H}_{6} \mathrm{~N}_{2} \mathrm{~S} \\
& M_{r}=150.21 \\
& \text { Monoclinic, } P 2_{1} / m \\
& \text { Hall symbol: }-\mathrm{P} 2 \mathrm{yb} \\
& a=4.915(1) \AA \\
& b=8.5590(17) \AA \\
& c=8.2920(17) \AA \\
& \beta=91.76(3)^{\circ} \\
& V=348.66(12) \AA^{3} \\
& Z=2
\end{aligned}
$$

Data collection

Enraf-Nonius CAD-4 diffractometer

Radiation source: fine-focus sealed tube Graphite monochromator $\omega / 2 \theta$ scans

Absorption correction: $\psi$ scan

(North et al., 1968)

$T_{\min }=0.896, T_{\max }=0.963$

903 measured reflections

\section{Refinement}

Refinement on $F^{2}$

Least-squares matrix: full

$R\left[F^{2}>2 \sigma\left(F^{2}\right)\right]=0.049$

$w R\left(F^{2}\right)=0.152$

$S=1.00$

813 reflections

45 parameters

0 restraints

Primary atom site location: structure-invariant direct methods
$F(000)=156$

$D_{\mathrm{x}}=1.431 \mathrm{Mg} \mathrm{m}^{-3}$

Mo $K \alpha$ radiation, $\lambda=0.71073 \AA$

Cell parameters from 25 reflections

$\theta=10-14^{\circ}$

$\mu=0.38 \mathrm{~mm}^{-1}$

$T=294 \mathrm{~K}$

Block, colorless

$0.30 \times 0.20 \times 0.10 \mathrm{~mm}$

813 independent reflections

647 reflections with $I>2 \sigma(I)$

$R_{\text {int }}=0.044$

$\theta_{\max }=27.0^{\circ}, \theta_{\min }=2.5^{\circ}$

$h=0 \rightarrow 6$

$k=0 \rightarrow 10$

$l=-10 \rightarrow 10$

3 standard reflections every $120 \mathrm{~min}$ intensity decay: $1 \%$

Secondary atom site location: difference Fourier map

Hydrogen site location: inferred from neighbouring sites

$\mathrm{H}$-atom parameters constrained

$w=1 /\left[\sigma^{2}\left(F_{\mathrm{o}}{ }^{2}\right)+(0.1 P)^{2}+0.059 P\right]$

where $P=\left(F_{\mathrm{o}}^{2}+2 F_{\mathrm{c}}^{2}\right) / 3$

$(\Delta / \sigma)_{\max }<0.001$

$\Delta \rho_{\max }=0.37{\mathrm{e} \AA^{-3}}^{-3}$

$\Delta \rho_{\min }=-0.26{\mathrm{e} \AA^{-3}}^{-3}$

Special details

Geometry. All e.s.d.'s (except the e.s.d. in the dihedral angle between two 1.s. planes) are estimated using the full covariance matrix. The cell e.s.d.'s are taken into account individually in the estimation of e.s.d.'s in distances, angles and torsion angles; correlations between e.s.d.'s in cell parameters are only used when they are defined by crystal symmetry. An approximate (isotropic) treatment of cell e.s.d.'s is used for estimating e.s.d.'s involving 1.s. planes.

Refinement. Refinement of $F^{2}$ against ALL reflections. The weighted $R$-factor $w R$ and goodness of fit $S$ are based on $F^{2}$, conventional $R$-factors $R$ are based on $F$, with $F$ set to zero for negative $F^{2}$. The threshold expression of $F^{2}>\sigma\left(F^{2}\right)$ is used only for calculating $R$-factors(gt) $e t c$. and is not relevant to the choice of reflections for refinement. $R$-factors based on $F^{2}$ are statistically about twice as large as those based on $F$, and $R$ - factors based on ALL data will be even larger.

Fractional atomic coordinates and isotropic or equivalent isotropic displacement parameters $\left(\AA^{2}\right)$

\begin{tabular}{lllll}
\hline & $x$ & $y$ & $z$ & $U_{\text {iso }} * / U_{\text {eq }}$ \\
\hline $\mathrm{S}$ & $0.06322(19)$ & 0.2500 & $0.88609(10)$ & $0.0510(3)$ \\
$\mathrm{N}$ & $-0.2841(4)$ & $0.1239(2)$ & $1.1022(2)$ & $0.0465(5)$ \\
$\mathrm{H} 0 \mathrm{~A}$ & -0.2505 & 0.0281 & 1.0783 & $0.056^{*}$
\end{tabular}




$\begin{array}{lllll}\text { C1 } & -0.7826(5) & 0.1687(4) & 1.4250(3) & 0.0644(7) \\ \text { H1A } & -0.8914 & 0.1154 & 1.4964 & 0.077^{*} \\ \text { C2 } & -0.6229(5) & 0.0844(3) & 1.3201(3) & 0.0561(7) \\ \text { H2A } & -0.6243 & -0.0242 & 1.3195 & 0.067^{*} \\ \text { C3 } & -0.4611(4) & 0.1684(3) & 1.2162(3) & 0.0437(5) \\ \text { C4 } & -0.1646(7) & 0.2500 & 1.0292(4) & 0.047\end{array}$

Atomic displacement parameters $\left(\AA^{2}\right)$

\begin{tabular}{lllllll}
\hline & $U^{11}$ & $U^{22}$ & $U^{33}$ & $U^{12}$ & $U^{13}$ & $U^{23}$ \\
\hline $\mathrm{S}$ & $0.0671(6)$ & $0.0270(5)$ & $0.0588(6)$ & 0.000 & $0.0015(4)$ & 0.000 \\
$\mathrm{~N}$ & $0.0585(11)$ & $0.0227(9)$ & $0.0578(12)$ & $-0.0010(8)$ & $-0.0061(9)$ & $0.0009(8)$ \\
$\mathrm{C} 1$ & $0.0621(14)$ & $0.0558(17)$ & $0.0755(18)$ & $-0.0084(13)$ & $0.0082(13)$ & $0.0065(14)$ \\
$\mathrm{C} 2$ & $0.0681(15)$ & $0.0359(13)$ & $0.0640(16)$ & $-0.0047(12)$ & $-0.0032(13)$ & $0.0047(11)$ \\
$\mathrm{C} 3$ & $0.0484(11)$ & $0.0302(12)$ & $0.0519(13)$ & $0.0009(9)$ & $-0.0094(9)$ & $-0.0003(9)$ \\
$\mathrm{C} 4$ & 0.057 & 0.029 & 0.054 & 0.000 & -0.019 & 0.000 \\
\hline
\end{tabular}

Geometric parameters $\left(\AA,{ }^{\circ}\right)$

\begin{tabular}{llll}
\hline $\mathrm{S}-\mathrm{C} 4$ & $1.656(4)$ & $\mathrm{C} 1-\mathrm{H} 1 \mathrm{~A}$ & 0.9300 \\
$\mathrm{~N}-\mathrm{C} 3$ & $1.359(3)$ & $\mathrm{C} 2-\mathrm{C} 3$ & $1.390(3)$ \\
$\mathrm{N}-\mathrm{C} 4$ & $1.378(3)$ & $\mathrm{C} 2-\mathrm{H} 2 \mathrm{~A}$ & 0.9300 \\
$\mathrm{~N}-\mathrm{H} 0 \mathrm{~A}$ & 0.8600 & $\mathrm{C} 3-\mathrm{C} 3^{\mathrm{i}}$ & $1.398(4)$ \\
$\mathrm{C} 1-\mathrm{C} 2$ & $1.391(4)$ & $\mathrm{C} 4-\mathrm{N}^{\mathrm{i}}$ & $1.378(3)$ \\
$\mathrm{C} 1-\mathrm{C} 1^{\mathrm{i}}$ & $1.391(6)$ & & \\
$\mathrm{C} 3-\mathrm{N}-\mathrm{C} 4$ & $112.1(2)$ & $\mathrm{C} 1-\mathrm{C} 2-\mathrm{H} 2 \mathrm{~A}$ & 121.2 \\
$\mathrm{C} 3-\mathrm{N}-\mathrm{H} 0 \mathrm{~A}$ & 123.9 & $\mathrm{~N}-\mathrm{C} 3-\mathrm{C} 2$ & $132.6(2)$ \\
$\mathrm{C} 4-\mathrm{N}-\mathrm{H} 0 \mathrm{~A}$ & 123.9 & $\mathrm{~N}-\mathrm{C} 3-\mathrm{C} 3^{\mathrm{i}}$ & $106.27(12)$ \\
$\mathrm{C} 2-\mathrm{C} 1-\mathrm{C} 1^{\mathrm{i}}$ & $121.24(16)$ & $\mathrm{C} 2-\mathrm{C} 3-\mathrm{C} 3^{\mathrm{i}}$ & $121.11(15)$ \\
$\mathrm{C} 2-\mathrm{C} 1-\mathrm{H} 1 \mathrm{~A}$ & 119.4 & $\mathrm{~N}-\mathrm{C} 4-\mathrm{N}^{\mathrm{i}}$ & $103.2(3)$ \\
$\mathrm{C} 1^{\mathrm{i}}-\mathrm{C} 1-\mathrm{H} 1 \mathrm{~A}$ & 119.4 & $\mathrm{~N}-\mathrm{C} 4-\mathrm{S}$ & $128.40(15)$ \\
$\mathrm{C} 3-\mathrm{C} 2-\mathrm{C} 1$ & $117.6(2)$ & $\mathrm{N}-\mathrm{C} 4-\mathrm{S}$ & \\
$\mathrm{C} 3-\mathrm{C} 2-\mathrm{H} 2 \mathrm{~A}$ & 121.2 & & $-0.6(3)$ \\
& & $\mathrm{C} 1-\mathrm{C} 2-\mathrm{C} 3-\mathrm{C} 3^{\mathrm{i}}$ & $-1.5(3)$ \\
$\mathrm{C} 1^{\mathrm{i}}-\mathrm{C} 1-\mathrm{C} 2-\mathrm{C} 3$ & $0.6(3)$ & $\mathrm{C} 3-\mathrm{N}-\mathrm{C} 4-\mathrm{N}^{\mathrm{i}}$ & $179.5(2)$ \\
$\mathrm{C} 4-\mathrm{N}-\mathrm{C} 3-\mathrm{C} 2$ & $-179.1(2)$ & $\mathrm{C} 3-\mathrm{N}-\mathrm{C} 4-\mathrm{S}$ & \\
$\mathrm{C} 4-\mathrm{N}-\mathrm{C} 3-\mathrm{C} 3^{\mathrm{i}}$ & $1.0(2)$ & & \\
$\mathrm{C} 1-\mathrm{C} 2-\mathrm{C} 3-\mathrm{N}$ & $179.5(2)$ & & \\
& & & \\
\hline
\end{tabular}

Symmetry code: (i) $x,-y+1 / 2, z$.

Hydrogen-bond geometry $\left(\AA,{ }^{\circ}\right)$

\begin{tabular}{lllll}
\hline$D-\mathrm{H} \cdots A$ & $D-\mathrm{H}$ & $\mathrm{H} \cdots A$ & $D \cdots A$ & $D-\mathrm{H} \cdots A$ \\
\hline $\mathrm{N}-\mathrm{H} 0 A \cdots \mathrm{S}^{\mathrm{ii}}$ & 0.86 & 2.57 & $3.3798(19)$ & 158 \\
\hline
\end{tabular}

Symmetry code: (ii) $-x, y-1 / 2,-z+2$. 